\title{
Bioavailability and metabolism of phenolic compounds from wholegrain wheat and
} aleurone-rich wheat bread.

Letizia Bresciani ${ }^{1,2}$, Francesca Scazzina ${ }^{1}$, Roberto Leonardi ${ }^{3,4}$, Elisabetta Dall'Aglio ${ }^{3}$, Michael Newell ${ }^{5,6}$, Margherita Dall'Asta ${ }^{1,2}$, Camilla Melegari ${ }^{7}$, Sumantra Ray ${ }^{5,6}$, Furio Brighenti ${ }^{1}$, Daniele Del Rio ${ }^{1,2,5^{*}}$.

${ }^{1}$ The Laboratory of Phytochemicals in Physiology, Human Nutrition Unit, Department of Food Science, University of Parma, 43125 Parma, Italy

${ }^{2}$ LS9 Bioactives and Health, Interlab Group, Department of Food Science, University of Parma, Parma, Italy

${ }^{3}$ Department of Clinical and Experimental Medicine, University of Parma, 43125 Parma, Italy ${ }^{4}$ Department of Nutritional Rehabilitation, Policlinico San Pietro, Ponte San Pietro (Bergamo), Gruppo San Donato (GSD), Italy

${ }^{5}$ The Need for Nutrition Education/Innovation Programme (NNEdPro), University of Cambridge

${ }^{6}$ Medical Research Council (MRC) Human Nutrition Research (HNR), Cambridge ${ }^{7}$ Barilla G. e R. F.lli, Via Mantova, 166, IT-43122 Parma, Italy

Received: $15 / 03 / 2016$

Revised: 25/05/2016;

Accepted: 29/05/2016

This article has been accepted for publication and undergone full peer review but has not been through the copyediting, typesetting, pagination and proofreading process, which may lead to differences between this version and the Version of Record. Please cite this article as doi: 10.1002/mnfr.201600238.

This article is protected by copyright. All rights reserved. 
*Correspondence: Dr. Daniele Del Rio, Department of Food Science - Human Nutrition Unit, Building C, Via Volturno 39, 43125, Parma (PR), Italy. Tel: +39 0521 903830. E-mail: daniele.delrio@unipr.it

Keywords: aleurone/phenolic bioavailability/wholegrain

\section{ABSTRACT}

Scope: This work aimed at investigating absorption, metabolism and bioavailability of phenolic compounds after consumption of wholegrain bread or bread enriched with an aleurone fraction.

Methods and results: Two commercially available breads were consumed by 15 participants on three occasions and matched for either the amount of ferulic acid in the bread or the amount of bread consumed. Urine was collected for $48 \mathrm{~h}$ from all the volunteers for phenolic metabolite quantification. Blood samples were collected for $24 \mathrm{~h}$ following bread consumption in 5 participants. A total of 12 and 4 phenolic metabolites were quantified in urine and plasma samples, respectively. Metabolites were sulfate and glucuronic acid conjugates of phenolic acids, and high concentrations of ferulic acid-4'-O-sulfate, dihydroferulic acid-4'-O-sulfate and dihydroferulic acid-O-glucuronide were observed. The bioavailability of ferulic acid was significantly higher from the aleurone-enriched bread when all ferulic acid metabolites were accounted for.

Conclusions: The study shows that low amounts of aleurone-enriched bread resulted in equivalent plasma levels of ferulic acid as wholegrain bread. This could suggest that, if the absorbed phenolic metabolites after wholegrain product intake exert health benefits, equal 
levels could be reached through the consumption of lower doses of refined products

enriched in aleurone fraction.

The study shows that low amounts of bread enriched in aleurone, the metabolically active innermost part of the bran, resulted in equivalent plasma levels of ferulic acid as wholegrain bread. This could suggest that, if the absorbed phenolic metabolites after wholegrain product intake exert health benefits, equal levels could be reached through the consumption of lower doses of refined products enriched in aleurone fraction.

\section{INTRODUCTION}

Increasing epidemiological, mainly observational, evidence suggests that wholegrain cereal consumption is linked to a reduction in the risk of chronic and degenerative diseases such as type 2 diabetes, cardiovascular disease, obesity, and cancer [1-4]. Although the mechanisms proposed for potential preventative effects have not been elucidated and remain speculative, the amounts of dietary fiber, vitamins, minerals and phytochemicals in wholegrain cereal appear to have key roles [5]. Inflammation might mediate the process, as suggested by the effect of wholegrain cereals on inflammatory markers such as C-reactive protein (CRP), TNF- $\alpha$, plasminogen activator inhibitor-1 and interleukin (IL)-6 [6-9]. Despite the evidence and the high levels of bioactive nutrients and non-nutrients, wholemeal cereal consumption remains lower than recommended in guidelines. In the United States, the consistent promotion of wholegrain products led to a $23.4 \%$ rise in consumption from 2008 to 2010 . However, the present average intake of $14 \mathrm{~g} /$ day is still considerably lower than the recommended intake of at least $48 \mathrm{~g} /$ day [10]. Whole-wheat is the main wholegrain cereal consumed in Western countries and represents an important source of dietary fiber [11]; minerals such as potassium, phosphorus, magnesium; vitamins, mainly niacin and folate; and phenolic compounds [12]. Phenolic 
compounds in wheat bran include primarily hydroxycinnamic acids among which ferulic acid is the most abundant representing the 70 to $90 \%$, caffeic acid, $p$-coumaric acid, sinapic acid, and benzoic acid derivatives, including protocatechuic acid, $p$-hydroxybenzoic acid, salicylic acid, vanillic acid; and syringic acid $[12,13]$. The whole-wheat kernel is usually processed to obtain the bran fraction which contains $45-50 \%$ of the aleurone layer. The aleurone layer is a metabolically active monolayer that is the innermost part of the bran and represents $5-8 \%$ of the wheat kernel $[12,14]$. The aleurone layer is usually removed during milling for its firm attachment to the external integuments [15]. However, the aleurone has a high content of fiber, protein, minerals, phytates, B vitamins (niacin, riboflavin and folate), lipidic compounds, plant sterols, and phenolic compounds [12]. Moreover, being protected from the environment, its content of pollutants such as pesticide residues, aflatoxins and heavy metals is reduced compared to the pericarp [16]. Due to its high content in nutrients and low level of contaminants, the development of new techniques to separate the aleurone from other bran layers has been actively sought by the food industry [16]. Most phenolic acids exist in the aleurone layer and in the wholegrain in three forms; soluble free acids; soluble conjugated moieties esterified to sugars or other low molecular weight compounds; and insoluble bound moieties that are esterified to the fiber fraction, mainly arabinoxylans. Ferulic acid, in particular, occurs partly in the free glycosylated form, but principally ( 92\%) covalently esterified with plant cell wall polysaccharides by $0-5$ position linkage $[11,17]$. Moreover, ferulic acid undergoes dimerization in planta to form oligomers, namely dihydrodiferulic acids (ferulic acid dimers) and ferulic trimers $[18,19]$. The linkage of hydroxycinnamates with the fiber fraction reduces the bioaccessibility [13] and bioavailability [20] of the phenolic fraction. Although, recently proposed technological 
bioprocessing demonstrated the capacity to improve ferulic bioaccessibility $[13,21]$, it is necessary to consider the complex set of transformations that cereal phenolics are subjected to within the human body following ingestion.

Several studies have demonstrated that phenolic compounds are extensively metabolized and heavily modified after ingestion. After polyphenol-rich food intake, circulating phase-II and microbiota-derived catabolites are predominant, whereas native compounds rarely appear in circulation. Minor chemical modifications in the small intestine include the formation of aglycones that undergo conjugation by phase II enzymes at the enterocyte, in the liver and at the tissue level after absorption [22, 23]. In contrast, a substantial amount of unmodified native compounds reach the colon where they are subjected to microbial catabolism that results in structurally modified catabolites (23). These catabolites are then absorbed through the portal circulation and undergo phase II metabolism [24]. Generally, the phenolic-fiber linkage in wholegrain products prevents almost all the phenolics from upper intestine absorption. The vast majority of these molecules reach the colon where the gut microbiota $\beta$-glucosidase and esterase activity permits a slow and continuous release of bound phenolics and ferulic acid [25]. The continuous release causes prolonged metabolite circulation in the bloodstream, which could explain the health benefits correlated with sustained wholegrain consumption [11]. Building on previous in vitro $[26,27]$ and animal studies [28] performed by our group the aim of this investigation was to assess the human absorption, metabolism and bioavailability of phenolic compounds following the consumption of whole-wheat. We also aim to measure and compare the concentration of metabolites in urine and plasma following the consumption of wholegrain bread or bread enriched with aleurone fraction. 


\section{MATERIALS AND METHODS}

\subsection{Participants}

Fifteen participants were recruited to take part in the study. Selected participants were 8 males and 7 females, $26 \pm 4$ years old, $62.4 \pm 13.5 \mathrm{~kg}, 1.7 \pm 0.1 \mathrm{~m}$ and $21 \pm 3 \mathrm{~kg} / \mathrm{m}^{2}$ (data expressed as mean \pm SD). Exclusion criteria included: not being pregnant or nursing; not being diagnosed with gluten intolerance or metabolic disorders such as hypertension, dyslipidemia, glucose intolerance or diabetes; non users of vitamin/mineral/micronutrient supplementation, and not taking any medication/drugs or antibiotic therapy. All participants were informed about the purpose of the study and provided written informed consent before their inclusion in the trial. Relevant principles of Good Clinical Practice were followed throughout the study. The study was approved by the Ethics Committee for Parma Hospital and University and registered on ClinicalTrials.gov, identification number: NCT02353234. A

101 flow diagram of the progress through the phases of the trial is reported in supporting 102 information figure 1.

\subsection{Study design}

105 In a single-blind, randomized, crossover study design two commercially available breads 106 (Barilla S.P.A.) were consumed by each participant on three separate occasions. The 107 phenolic composition and concentrations of the two breads were first confirmed [27] prior 108 to meal portion calculation. The nutritional values and the ferulic acid content per portion are reported in Table 1. 
Each participant was tested under three acute treatments that differed for the amount of

111 ferulic acid or the bread quantity consumed. Each acute feeding experiment was separated

112 by a minimum 1-week washout period. The bread products consumed consisted of: WGB -

$11394 \mathrm{~g}$ of wholegrain bread containing approximately $87 \mathrm{mg}$ of total ferulic acid; AB-94 - $94 \mathrm{~g}$

114 of a commercial wheat bread enriched in aleurone fraction $(6 \% \mathrm{w} / \mathrm{w}$ of the inner part of

115 aleurone [26]) containing approximately $43 \mathrm{mg}$ of total ferulic acid, which matches WGB for

116 total bread consumed; AB-190 - $190 \mathrm{~g}$ of a commercial bread enriched in aleurone fraction

117 (6\% w/w of the inner part of aleurone [26]), containing approximately $87 \mathrm{mg}$ of total ferulic

118 acid, which matches WGB for the total amount of ingested ferulic acid.

119 Participants were asked to consume a polyphenol free diet two days prior to and two days

120 after laboratory visits. A list of permitted and forbidden foods was supplied to facilitate

121 participant adherence to the dietary restrictions. Additionally, a food diary was provided to record all meals and to check for compliance to the recommended diet. During the

123 laboratory visits participants collected their urine following fasting $\left(\mathrm{t}_{0}\right)$, and after product

124 consumption at: $0-3,3-6,6-10,10-14,14-24,24-28,28-34$ and $34-48 \mathrm{~h}$. The urine volumes

125 were recorded and two aliquots of $2 \mathrm{~mL}$ each were stored at $-80^{\circ} \mathrm{C}$ until analysis. Five

126 randomly selected participants provided $3 \mathrm{~mL}$ blood samples taken at fasting $\left(\mathrm{t}_{0}\right)$ and at 30

$127 \min , 1 \mathrm{~h}, 2 \mathrm{~h}, 4 \mathrm{~h}, 7 \mathrm{~h}$, and $24 \mathrm{~h}$ following bread consumption. Blood was collected in $\mathrm{K}_{2}$ EDTA

128 tubes, centrifuged for $15 \mathrm{~min}$ at $4,000 \mathrm{rpm}$ and $\approx 1.2 \mathrm{~mL}$ of plasma was separated and

129 stored at $-80{ }^{\circ} \mathrm{C}$ until analysis. The study design is shown in Fig. 1. 
All chemicals and solvents were of analytical grade. All solvents and reagents were purchased from Sigma-Aldrich (St. Louis, MO, USA). Vanillic acid (3-methoxy-4hydroxybenzoic acid), 3-hydroxybenzoic, 4- hydroxybenzoic, protocatechuic acid (3,4dihydroxybenzoic acid), 3',4'-dihydroxyphenylacetic acid, 3-(3'-hydroxyphenyl)propionic acid, 3-(4'-hydroxyphenyl)propionic acid, hippuric acid, 4-hydroxyhippuric acid, and ferouloylglycine were purchased from Sigma-Aldrich (St. Louis, MO, USA). Ferulic acid-4'-Osulphate disodium salt, isoferulic acid-3'-O-B-D-glucuronide, dihydroferulic acid 4'-O-sulfate

139

140

141

142 sodium salt, dihydroisoferulic acid 3-O-6-D-Glucuronide, and dihydrocaffeic acid-3'-Osulphate sodium salt were purchased from Toronto Research Chemicals (Toronto, Ontario, Canada). Ultrapure water from MilliQsystem (Millipore, Bedford, MA, USA) was used throughout the experiment.

\subsection{Urine and plasma extraction}

Urine samples were centrifuged at $14,000 \mathrm{rpm}$ for $10 \mathrm{~min}$ and $700 \mu \mathrm{L}$ of supernatant was diluted 1:2 (v/v) adding acidified water ( $0.1 \%$ formic acid $v / v)$. Samples were then filtered through a $0.45 \mu \mathrm{m}$ nylon filter before analysis.

Plasma samples were extracted for analysis following the method previously reported by Renouf [29]. Briefly, $400 \mu \mathrm{L}$ of plasma was added to $1.2 \mathrm{~mL}$ of ethanol. Samples were vortexed for $5 \mathrm{~min}$, centrifuged at 14,000 rpm for $10 \mathrm{~min}$ and $1.3 \mathrm{~mL}$ of supernatant was dried under a rotary-vacuum evaporation. The pellet was re-suspended with $65 \mu \mathrm{L}$ of acidified water ( $1 \%$ formic acid)/methanol $(50: 50, \mathrm{v} / \mathrm{v}$ ) and samples were vortexed again to dissolve the pellet and finally centrifuged at $14,000 \mathrm{rpm}$ for $5 \mathrm{~min}$ prior to analysis. 


\section{5 uHPLC/MS ${ }^{n}$ Analysis}

157 Urine and plasma samples were analyzed by ultra-high performance liquid chromatography coupled with mass spectrometry (UHPLC/MS) to identify and quantify the phenolic metabolites after bread consumption. An Accela ultra-high performance liquid chromatography (UHPLC) 1250 apparatus equipped with a linear ion trap mass spectrometer (LIT-MS) (LTQ XL, Thermo Fisher Scientific Inc., San Jose, CA, USA) fitted with a heated-ESI (H-ESI-II) probe (Thermo Fisher Scientific Inc.) was used. Compound separations were performed with a XSELECT ${ }^{\mathrm{TM}}$ HSS PFP column $(50 \times 2.1 \mathrm{~mm}), 2.5 \mu \mathrm{m}$ particle size (Waters, USA). A preliminary analysis was carried out in full scan, data-dependent $\mathrm{MS}^{3}$ mode, scanning from a mass to charge $(\mathrm{m} / \mathrm{z})$ of 100 to 800 to perform a preliminary assessment of the main phenolic metabolites present in the samples. On the basis of the obtained information, a second preliminary analysis was carried out in full $\mathrm{MS}^{2}$ and $\mathrm{MS}^{3}$ mode to confirm the identification of the detected metabolites and to record the retention time. Finally, quantification of all metabolites in biological samples was achieved using the single reaction monitoring (SRM) mode. The analyses were carried out in negative ionization

171 mode, with a capillary temperature of $275^{\circ} \mathrm{C}$, while the source was set at $250^{\circ} \mathrm{C}$. The sheath 172 gas flow was 40 units, while auxiliary and sweep gases were set to 5 units. The source 173 voltage was $3 \mathrm{kV}$. The capillary voltage and tube lens were -9 and $-53 \mathrm{~V}$, respectively. For chromatographic analysis, the mobile phase, pumped at a flow rate of $0.3 \mathrm{~mL} / \mathrm{min}$, was composed of acetonitrile containing $0.1 \%$ formic acid (solvent $A$ ) and $0.1 \%$ formic acid in water (solvent B). A linear gradient bringing A from 5 to $50 \%$ over 6.5 min was carried out for separation. All metabolites were fragmented using a collision-induced dissociation (CID) 
of 30 , using helium gas for the collision. Where available, phenolic acid catabolites were quantified using a calibration curve prepared with the standard compound. When not available, the conjugated metabolites were quantified with the most structurally similar compound (Table 2).

\subsection{Statistical analysis and absorption kinetic parameters}

Based on available published data [30], using an F test and an ANOVA test with repeated measures, it was estimated that 12 participants in each treatment group would give sufficient power ( $\alpha$ error of $0.05,80 \%$ power) to detect a significant difference of $5.0 \mu \mathrm{mol}$ of excreted ferulic acid.

Urine and plasma data were analyzed with a statistical software (SPSS, Chicago, IL, USA, version 21). All data were expressed as mean values \pm SEM. A general linear model repeated measures (ANOVA, LSD post-hoc test) analysis was performed to compare each metabolite among the three treatments. Statistical significance was set at $p<0.05$. Kinetic parameters of the plasmatic metabolites detected after bread consumption, including area under curve (AUC), plasma maximum concentration $\left(C_{\max }\right)$, time to reach the maximum plasma concentration ( $\left.T_{\max }\right)$, and, when available, apparent half-life $\left(T_{1 / 2}\right)$ were calculated by means of pharmacokinetic functions for Microsoft Excel. Data on pharmacokinetic parameters were presented as mean values \pm SEM.

\section{RESULTS}

All participants consumed the control bread (wholegrain bread) once and the breads enriched in aleurone fraction twice for a total of three experimental trials. The portion of 
201

202

203

204

205

206

207

208

209

210

211

212

213

214

215

216

217

218

219

220

221

222

each meal was calculated to ensure that the same dose of ferulic acid (WGB vs AB-190) or

the same quantity of bread (WGB vs AB-94) was administered. This approach allowed us to determine the bioavailability of ferulic acid in wholegrain products compared to aleuronerich bread, and to test the possible influence of the consumed dose on the phenolic acid absorption and metabolism. All participants consumed the entirety of provided meals, which were well tolerated.

Using LC-MS analysis, 12 metabolites were identified, or tentatively identified, and quantified in urine samples, whereas only 4 metabolites were quantifiable in plasma samples. The spectrometric characteristics of the detected catabolites are reported in Table 3. The phenolic composition of the tested products were mostly free or bound phenolic acids [27] with no parent compound detected in the analyzed urine and plasma samples

\subsection{Identification and Quantification of Urinary Metabolite}

Among the 12 quantified urinary metabolites, 7 compounds were detected as sulfate conjugates and one as a glucuronide conjugate, highlighting the importance of the interaction of native phenolic compounds with intestinal and hepatic sulfotransferases (SULTs) and uridine-5'-diphosphate glucuronosyltransferases (UGTs). The presence of only one glucuronidated metabolite could suggest a minor interaction of phenolic acids of wholegrain and aleurone fractions with UGTs, compared with SULTs [28]. Besides the sulfate and glucuronidated forms, one metabolite was detected in its free, aglyconic form, and three compounds were found as nitrogen-containing conjugates, a reaction strictly linked to microbial metabolism [31]. 
The most abundant metabolites in terms of cumulative excretion appeared to be hippuric acid and hydroxyhippuric acid. Two isomers of hydroxyhippuric acid were detected in all samples, but they were quantified together using the available standard. These nitrogencontaining metabolites were monitored due to their possible origin from phenolic compounds. However, the initial amount and the trend of the excretion curves suggests several potential origins as previously reported [23] (Supporting information Fig. 2). Since ferulic acid is the main phenolic compound, both in wholegrain and in aleurone-rich bread, high concentrations of ferulic acid metabolites (ferulic acid-4'-O-sulfate, dihydroferulic acid-4'-O-sulfate and dihydroferulic acid-O-glucuronide) were recovered in urine samples (Fig. 2). If the ferulic acid-4'-O-sulfate (Fig. 2a) indicates an interaction of the native compound with the SULTs, the dihydroferulic metabolites (Fig. $2 \mathrm{~b}$ and $2 \mathrm{c}$ ) provides evidence of a microbial action on the double $\mathrm{C}-\mathrm{C}$ bond prior to hepatic sulfation or glucuronidation. Colonic catabolism also resulted in the production of feruloylglycine [32] (Fig. 2d). Fig. 3 shows the cumulative excretion of dihydrocaffeic acid sulfate (Fig. 3a) and sinapic acid sulfate (Fig. 3b), with the latter tentatively identified due to the lack of a commercial standard. As previously reported for ferulic acid colonic metabolites, the importance of the microbiota on the biotransformation of native phenolic compounds was demonstrated by the presence of dihydrocaffeic acid sulfate and other low molecular weight metabolites such as vanillic acid-4-O-sulfate (Fig. 3c) and hydroxybenzoic acid sulfate (Fig. 3d), and of a negligible amount of 3-(phenyl)propionic acid-O-sulfate (Supporting information Fig. 2c). These metabolites could originate from all the native phenolic acids in the diet, not specifically to ferulic acid. For this reason, these specific metabolites cannot be taken into account when ferulic acid bioavailability is evaluated. Finally, $3^{\prime}, 4^{\prime}-$ 
dihydroxyphenylacetic acid was the only identified unconjugated compound (Supporting information Fig. 2d). The concentration in urine appeared to increase throughout the $48 \mathrm{~h}$ sampling period with no noticeable difference across the products ingested. By observing the slopes of the 48-h cumulative curves, the metabolic origin of each metabolite could be interpreted. Specifically, a steep slope recorded within the 0-3 $\mathrm{h}$ time period is suggestive of early absorption in the small intestine, and of a subsequent conjugation at the enterocyte or hepatic level. Hence, ferulic acid-4'-O-sulfate was the only metabolite derived from this metabolic path, indicating that the native compound could be partially absorbed in the small intestine (Fig. 2a). An increase in the slope at 3-6 h indicates a release of the most accessible fiber-linked-phenolic compounds by microbiota enzymatic activity. The release of native compounds in the colon led to increased absorption and further conjugation. For example, ferulic acid-4'-O-sulfate (Fig. 2a), sinapic acid sulfate (Fig. 3b) and hydroxybenzoic acid sulfate (Fig. 3b) curves exhibited a increased rate of appearance within 3 and $6 \mathrm{~h}$. Finally, increasing slopes detected from 6 to $48 \mathrm{~h}$ explain a deeper microbiota catabolism. In particular, metabolites excreted during these excretion periods could be associated with i) a long transient time in the large intestine, according to normal metabolism of dietary fiber-rich products [33], which caused a slower release of less accessible fiber-linked phenolics; ii) the microbial enzymatic activity needed to convert the released phenolic compounds into lower molecular weight molecules (as vanillic acid, hydroxybenzoic acid (Fig. 3c and d), 3-(phenyl)propionic acid (Supporting information Fig. 2c)), or into nitrogen-containing conjugates (hippuric acid, hydroxyhippuric acid (Supporting information Fig. 2a and 2b) and feruloylglycine (Fig. 2d)), or into reduced forms 
(dihydrocaffeic acid (Fig. 3a) and dihydroferulic acid (Fig. 2b and 2c)); or iii) the hepatic SULTs and UGTs activity on phenolics absorbed from the colon.

Observation of the 48-h urinary excretion (Fig. 2, 3 and Supporting information Fig. 2) suggests that the consumption of AB-190 (bread enriched with aleurone fraction that contained the same dose of ferulic acid of the wholegrain bread) is generally linked to higher excretion of almost all the detected metabolites, with peculiar differences for feruloylglycine, hippuric acid, dihydrocaffeic acid sulfate, and sinapic acid sulfate.

\subsection{Bioavailability}

To calculate the bioavailability of specific wheat phenolics, we have computed the ratio between the total metabolite $48 \mathrm{~h}$ urinary excretion and the total intake of parent compounds through the three fed bread portions.

Although the 48-h cumulative excretion of dihydrocaffeic acid sulfate and sinapic acid sulfate appeared significantly higher after the consumption of the aleurone enriched bread, the bioaccessibility and bioavailability of caffeic or sinapic acid cannot be quantified as the portion meal was not standardized for their respective contents.

On the contrary, the bioavailability of ferulic acid can be evaluated by focusing specifically on its direct metabolites, such as ferulic acid-4'-O-sulfate, dihydroferulic acid-4'-O-sulfate and dihydroferulic acid-O-glucuronide, and by correlating their total amount to the amount of native free and bound ferulic acid provided through each test meal. Fig. 4 shows that the bioavailability of ferulic acid in aleurone enriched bread containing the same dose of ferulic acid (AB-190) was slightly higher than after the control wholegrain product, but this difference did not reach a statistical significance. On the contrary, the bioavailability of 
291

ferulic acid in bread (AB-94) enriched in aleurone fraction consumed in same quantity as wholegrain bread was significantly higher.

\subsection{Plasma kinetics of phenolic compounds}

The second target of the study focused on plasma pharmacokinetics of phenolic compounds in bread. Contrary to urinary data, only 4 metabolites were detected in quantifiable concentrations in plasma samples. Hippuric acid and hydroxyhippuric acid (where the concentration was calculated considering both of the isomers) had the highest concentration among all circulating metabolites (Supporting information Fig. 3a and b). Although hippuric acid exhibited a peak plasma concentration at $7 \mathrm{~h}$, indicating a possible colonic degradation of native phenolic compounds, the absorption curves appeared unclear and these metabolites could not be directly associated with phenolic acid metabolism. On the contrary, the presence of ferulic acid-4'-O-sulfate and dihydroferulic acid-4'-O-sulfate can be strictly linked to bread consumption (Fig. 5). The curve reported for ferulic acid-4'-Osulfate (Fig. 5a) clearly demonstrates the absorption and the rapid conjugation of the native ferulic acid in the first part of the gastrointestinal tract, confirming what was observed in urines. The evidence was supported by the calculated $T_{\max }$ value close to $1 \mathrm{~h}$. The circulating concentrations rapidly decreased within $3 \mathrm{~h}$ and progressively until $24 \mathrm{~h}$. Although the curve derived from the consumption of the product enriched in aleurone fraction containing less ferulic acid (AB-94) showed a lower $C_{\max }$ and $A U C$ s value, no significant differences were observed in the kinetic parameters among the three products. The dihydroferulic acid-4'-Osulfate absorption curve (Fig. 5b) distinctly outlined a maximum peak at $7 \mathrm{~h}$, which was higher after the consumption of AB-190, in accordance with its colonic formation [34], and 
its concentration decreased before the last collection time point. However, because of the lack of intermediate time points between $7 \mathrm{~h}$ and $24 \mathrm{~h}$ it was impossible to exclude the

316 presence of additional peak concentrations within this period.

\section{DISCUSSION}

319 The present study compared, for the first time, the in vivo human absorption, metabolism and bioavailability of phenolic compounds present in two types of bread: wholegrain bread and wheat bread enriched in aleurone fraction. We standardized bread intake based on the dose of the main phenolic acid, ferulic acid, and accounted for free and bound phenolics, as

323 well as ferulic acid oligomers [27].

324 Previous research has compared the in vitro early digestion of wholegrain bran to the

325 aleurone fraction. Zaupa and colleagues reported the in vitro bioaccessibility of the main

326 phenolic compounds were higher in the aleurone flour compared to unfractionated bran

327 [26]. These results were corroborated when the same approach was used to measure the in 328 vitro bioaccessibility of phenolic compounds in wholegrain flour bread or aleurone-rich flour bread. All the main phenolic acids were significantly more bioaccessible in the bread enriched in aleurone fraction [27]. Despite small intestine bioaccessibility, it is important to assess colonic bioaccessibility, as phenolic compounds are poorly absorbed in the small

332 intestine [23]. In vitro colonic biotransformation has revealed the capacity of colonic microbiota enzymes to catabolize ferulic oligomers, to release fiber-linked compounds, and to convert them into simple metabolites, such as ferulic and dihydroferulic acid and other aromatic metabolites such as phenylpropionic, phenylacetic and benzoic acid derivatives 
$[18,35,36]$, microbial metabolites which have been reported to be efficiently absorbed at

337 large intestinal level [37].

338 Existing animal studies that fed a whole-wheat meal or aleurone-rich meal led to the

339 identification of both human and microbial metabolites that are mainly conjugated with

340 sulfate or glucuronic acid residues [28, 38, 39]. Calani and colleagues (2014) fed rats a

341

342

343

344

345

346

347

348

349

350

351

352

353

354

355

356

357

358

control pellet or a wheat aleurone-rich pellet. Fifteen metabolites were quantified and were mostly sulfate conjugated. Of these fifteen, six metabolites were detected in the present human in vivo study including: ferulic acid-4'-O-sulfate, dihydroferulic acid-4'-O-sulfate, vanillic acid-4-O-sulfate, 3-(phenyl)propionic acid-O-sulfate, $33^{\prime}, 4^{\prime}$-dihydroxyphenylacetic acid and hippuric acid. In the same rat feeding study the 24-h urinary recovery for the majority of metabolites was higher when the aleurone-rich pellet was consumed, demonstrating a higher absorption of aleurone phenolic compounds [28]. A better absorption of the phenolic acid of aleurone fraction rather than wholegrain was also established by Nørskov and colleagues (2013). Pigs that consumed aleurone-rich wheat flour for $72 \mathrm{~h}$ had a higher phenolic metabolite plasma concentration in comparison to a whole-wheat diet. However, the phenolic compound intake was not standardized between the two diets [39]. No human studies are available on aleurone phenolic acid metabolism and absorption. However, some human intervention studies that investigated wholegrain cereal consumption reported urinary excretion of ferulic acid conjugates, feruloylglycine and sinapic acid conjugates [40]. Additionally, plasma circulating concentrations of ferulic acid conjugates, vanillic acid, 3-(hydroxyphenyl)propionic acid conjugates, and sinapic acid conjugates $[21,40]$, have been reported as ranging from nanomolar to low micromolar. The absence of some plasma microbial catabolites following wholegrain bread intake in the 
present study could be explained by the lower dose of ferulic acid ( 3-3.5 fold lower respectively), than previously utilized in other studies.

In the present study we standardized ferulic intake between the wholegrain bread and the aleurone bread trials and found a total bioavailability of $4-8 \%$. The bioavailability of ferulic acid in humans was higher than in rats fed aleurone-rich pellets where it was approximately $0.7 \%$ [28]. In contrast, ferulic acid bioavailability was similar, at $4-5.5 \%$, when rats were fed a cereal-based meal [20]. Additionally, other human studies that examined whole-wheat bread or high-bran cereals report a $3-10 \%$ bioavailability of ferulic acid $[21,40]$. Other studies that report wholegrain ferulic acid bioavailability, with the exception of Calani et al (2014), do not consider the amount of ferulic acid dimers or trimers contained in the test meal.

As bioavailability could have been influenced both by the type of ferulic acid source (wholegrain flour or aleurone fraction) and by the amount of bread (with a possible bulk effect and/or absorption saturation), the chosen study design allowed us to discriminate between these two potential co-factors. When the same amount of ferulic acid was fed (87mg, but the two bread amounts were different), the bioavailability did not result significantly different between aleurone enriched and wholemeal breads (Fig.4), unless feruloyglycine was accounted for in the calculation (Supporting information Fig. 4). When, however, the same amount of bread was fed $(94 \mathrm{~g}$, but the total ferulic acid intake was different), ferulic acid from aleurone-enriched bread was significantly better absorbed and excreted. Nevertheless, it must be pointed out that the excretion of feruloylglycine in the bioavailability calculation could be misleading, since the concentration of this metabolite was detectable already at the beginning $\left(T_{0}\right)$ of each treatment, as also previously reported 
[40], suggesting that its $48 \mathrm{~h}$-cumulative excretion could result from the catabolism of other ingested or biotransformed compounds.

Plasma levels of ferulic acid metabolites were not significantly different after the three feedings, indicating that even with half dose of ferulic acid (introduced with AB-94), consumers could reach plasma levels of ferulic acid metabolites comparable to those obtained from a higher dose introduced through wholemeal bread or higher volumed of aleurone bread.

The higher ferulic bioavailability in bread enriched with the aleurone fraction compared to wholegrain bread could be explained by the different bran structure. Increased bioaccessibility, and hence bioavailability, of wheat aleurone phenolics has been demonstrated in the literature by applying different approaches, like enzymatic treatments targeting specific linkages in wheat bran $[35,41]$, fermentation systems, used as sources of specific enzymes $[21,41]$, or high energy grinding, increasing the specific surface of the bran fractions [16, 35]. Conversely, the higher bioavailability we observed following the lower ferulic consumption suggests a potential negative dose-metabolic response explained by a reduction in the capacity to metabolize and absorb phenolic acids caused by a higher level of intake. Future studies should focus on establishing the mechanisms involved in the absorption of phenolic acids from aleurone-rich products.

In recent years, the consumption of (poly)phenol-rich foods has been largely connected to beneficial health effects $[42,43]$. As such, evaluating the metabolism and bioavailability of phenolic acids (particularly ferulic acid) in widely consumed staple products, such as bread, becomes of particular interest. Additionally, more studies are appearing in the literature trying to unravel the mechanisms behind the protective effects exerted by wholegrain 
405

foods, with some of these highlighting the possible involvement of phenolic acids. For example, the circulating levels of specific phenolic metabolites detected after wholegrain consumption in humans, such as ferulic acid conjugates, vanillic acid and hydroxyphenylpropionic acid, have been specifically related to a reduction in pro / antiinflammatory cytokine ratios. These metabolites result in an immune-modulatory effect [21] and a reduction in TNF- $\alpha$ and an enhancement in IL-10 correlated to the improvement of the inflammation status [44]. Furthermore, two of the main phenolic acid metabolites $(3,4-$ dihydroxyphenylacetic acid and 3-hydroxyphenylpropionic acid) have been described in vitro to potentiate glucose-stimulated insulin secretion in a beta cell line INS-1E and in rat pancreatic islets [45]. Also, these metabolites are reported to protect against beta cell dysfunction and death induced by tert-butyl hydroperoxide, through the activation of protein kinase $\mathrm{C}$ and the extracellular regulated kinase pathways [45]. Moreover, the dihydroxylated microbial compound 3,4-dihydroxyphenylacetic acid, as well as 3,4dihydroxyphenylpropionic, exhibited anti-inflammatory properties, modulating the production of the main pro-inflammatory cytokines, as TNF- $\alpha$, IL-1 $\beta$ and IL-6, in LPSstimulated peripheral blood mononuclear cells. Contrarily, 4-hydroxyhippuric acid exerted its anti-inflammatory property only acting on TNF- $\alpha$ secretion [46]. Finally, very recently, Malunga and colleagues described ferulic acid and feruloylated arabinoxylan mono/oligosaccharides as possible inhibitors of sucrase and maltase functions of $\alpha$-glucosidase in Caco-2 and rat intestinal cells; moreover glucose uptake in Caco-2 cells was partially inhibited by their presence [47].

Limited evidence is available on potential human health benefits due to aleurone. Rats fed with wheat aleurone did not experience improvement of any parameter associated with 
obesity, such as body weight gain, adiposity, fasting blood glucose, plasma insulin and leptin, anti-inflammatory markers and oxidative stress markers [48]. Ounnas and colleagues (2014) demonstrated that plasma eicosapentaenoic acid concentration increased in rats fed with wheat aleurone, while docosahexaenoic acid and omega- 6 fatty acids were not affected [49]. In humans, the consumption of wheat aleurone-rich foods significantly lowered the plasma concentration of the inflammatory marker, C-reactive protein, which is an independent risk factor for CVD. No changes were observed in other markers of inflammation, antioxidant status or endothelial function [50]. However, it remains to be established whether the potential health benefits reported for wholegrain consumption are dependent on a specific component or a combination of components present in wholegrains and possibly in aleurone fractions.

In conclusion, this is the first human intervention study in which phenolic compound metabolism, absorption and bioavailability in wholegrain bread have been compared to those introduced through bread enriched with aleurone layer. The results of this study, besides showing a general increased bioavailability of ferulic acid in aleurone-rich bread, demonstrate that high levels of ferulic acid metabolites can be achieved in humans with the consumption of relatively low amounts (4 slices) of white, aleurone-enriched bread. We are only able to speculate as if the potential mechanisms behind the health effects linked to wholegrains are maintained with aleurone-rich food consumption. However, if the circulating phenolic metabolites after intake of wholegrain products are involved in physiological benefits [44], this study demonstrates that the same levels could be reached through consumption of refined products enriched in aleurone fractions. This study paves the way for future research focused on measuring the modulation of health related 
biomarkers by aleurone and its phenolics in both health individuals as well as those with

disease risk factors.

453 Supporting material

mnfr201600238-sup-0001-supporting_information_rev1_OK

We thank all the volunteers who participated in the study.

$L B, F S$ and DDR designed the research; $L B$ conducted the experiments, collected samples and

data, analyzed samples, drafted the manuscript; RL and EDA were medical study supervisors

and responsible for hematic sample collection; CM provided the bread products and

organized the portioning; $F S, M N, M D, S R$ and FB critically read and improved the protocol

and the manuscript; DDR secured the funding and has the primary responsibility for the final content; all authors: read and approved the final manuscript. CM is an employee of Barilla SpA. $L B, F S, R L, E D A, M N, M D, S R, F B$, and $D D R$ had no competing financial interests.

The study has been supported by Barilla S.p.A.

\section{REFERENCES}

466 [1] Lutsey, P. L., Jacobs, D. R., Jr., Kori, S., Mayer-Davis, E., et al., Whole grain intake and its cross467 sectional association with obesity, insulin resistance, inflammation, diabetes and subclinical CVD: 468 The MESA Study. Br J Nutr 2007, 98, 397-405.

469 [2] Ye, E. Q., Chacko, S. A., Chou, E. L., Kugizaki, M., Liu, S., Greater whole-grain intake is associated with lower risk of type 2 diabetes, cardiovascular disease, and weight gain. J Nutr 2012, 142, 13041313.

[3] Egeberg, R., Olsen, A., Loft, S., Christensen, J., et al., Intake of wholegrain products and risk of colorectal cancers in the Diet, Cancer and Health cohort study. Br. J. Cancer 2010, 103, 730-734. population-based case-control study in the San Francisco Bay Area, California. Am. J. Epidemiol. 2007, 166, 1174-1185. 
[6] Masters, R. C., Liese, A. D., Haffner, S. M., Wagenknecht, L. E., Hanley, A. J., Whole and refined grain intakes are related to inflammatory protein concentrations in human plasma. J Nutr 2010, 140, 587-594.

[7] Gaskins, A. J., Mumford, S. L., Rovner, A. J., Zhang, C., et al., Whole grains are associated with serum concentrations of high sensitivity C-reactive protein among premenopausal women. J Nutr 2010, 140, 1669-1676.

[8] Qi, L., van Dam, R. M., Liu, S., Franz, M., et al., Whole-grain, bran, and cereal fiber intakes and markers of systemic inflammation in diabetic women. Diabetes Care 2006, 29, 207-211.

[9] Jensen, M. K., Koh-Banerjee, P., Franz, M., Sampson, L., et al., Whole grains, bran, and germ in relation to homocysteine and markers of glycemic control, lipids, and inflammation 1. Am J Clin Nutr 2006, 83, 275-283.

[10] Sosland, L., Whole wheat flour production tops 20 million cwts: growth rate slows. Milling \& Baking News 2011, 26, 1, 29, 30, 32.

[11] Vitaglione, P., Napolitano, A., Fogliano, V., Cereal dietary fibre: a natural functional ingredient to deliver phenolic compounds into the gut. Trends in Food Science \& Technology 2008, 19, 451-463.

[12] Brouns, F., Hemery, Y., Price, R., Anson, N. M., Wheat aleurone: separation, composition, health aspects, and potential food use. Crit Rev Food Sci Nutr 2012, 52, 553-568.

[13] Mateo Anson, N., van den Berg, R., Havenaar, R., Bast, A., Haenen, G. R. M. M., Bioavailability of ferulic acid is determined by its bioaccessibility. Journal of Cereal Science 2009, 49, 296-300.

[14] Keaveney, E. M., Price, R. K., Hamill, L. L., Wallace, J. M., et al., Postprandial plasma betaine and other methyl donor-related responses after consumption of minimally processed wheat bran or wheat aleurone, or wheat aleurone incorporated into bread. Br J Nutr 2015, 113, 445-453.

[15] Lillioja, S., Neal, A. L., Tapsell, L., Jacobs, D. R., Whole grains, type 2 diabetes, coronary heart disease, and hypertension: Links to the aleurone preferred over indigestible fiber. Biofactors 2013, 39, 242-258.

[16] Hemery, Y. M., Anson, N. M., Havenaar, R., Haenen, G. R. M. M., et al., Dry-fractionation of wheat bran increases the bioaccessibility of phenolic acids in breads made from processed bran fractions. Food Research International 2010, 43, 1429-1438.

[17] Li, L., Shewry, P. R., Ward, J. L., Phenolic acids in wheat varieties in the HEALTHGRAIN Diversity Screen. J Agric Food Chem 2008, 56, 9732-9739.

[18] Braune, A., Bunzel, M., Yonekura, R., Blaut, M., Conversion of dehydrodiferulic acids by human intestinal microbiota. Journal of Agricultural and Food Chemistry 2009, 57, 3356-3362.

[19] Rondini, L., Peyrat-Maillard, M. N., Marsset-Baglieri, A., Fromentin, G., et al., Bound ferulic acid from bran is more bioavailable than the free compound in rat. J Agric Food Chem 2004, 52, 43384343.

[20] Adam, A., Crespy, V., Levrat-Verny, M. A., Leenhardt, F., et al., The bioavailability of ferulic acid is governed primarily by the food matrix rather than its metabolism in intestine and liver in rats. $J$ Nutr 2002, 132, 1962-1968.

[21] Mateo Anson, N., Aura, A. M., Selinheimo, E., Mattila, I., et al., Bioprocessing of wheat bran in whole wheat bread increases the bioavailability of phenolic acids in men and exerts antiinflammatory effects ex vivo. J Nutr 2011, 141, 137-143.

[22] Crozier, A., Jaganath, I. B., Clifford, M. N., Dietary phenolics: chemistry, bioavailability and effects on health. Nat. Prod. Rep. 2009, 26, 1001-1043.

[23] Del Rio, D., Rodriguez-Mateos, A., Spencer, J. P., Tognolini, M., et al., Dietary (poly)phenolics in human health: structures, bioavailability, and evidence of protective effects against chronic diseases. Antioxid Redox Signal 2013, 18, 1818-1892.

[24] Selma, M. V., Espin, J. C., Tomas-Barberan, F. A., Interaction between phenolics and gut microbiota: role in human health. J Agric Food Chem 2009, 57, 6485-6501.

This article is protected by copyright. All rights reserved. 
[25] Poquet, L., Clifford, M. N., Williamson, G., Transport and metabolism of ferulic acid through the colonic epithelium. Drug Metabolism and Disposition 2008, 36, 190-197.

[26] Zaupa, M., Scazzina, F., Dall'Asta, M., Calani, L., et al., In vitro bioaccessibility of phenolics and vitamins from durum wheat aleurone fractions. J Agric Food Chem 2014, 62, 1543-1549.

[27] Dall'Asta, M., Bresciani, L., Calani, L., Cossu, M., et al., In Vitro Bioaccessibility of Phenolic Acids from a Commercial Aleurone-Enriched Bread Compared to a Whole Grain Bread. Nutrients 2016, 8 , 42.

[28] Calani, L., Ounnas, F., Salen, P., Demeilliers, C., et al., Bioavailability and metabolism of hydroxycinnamates in rats fed with durum wheat aleurone fractions. Food Funct 2014.

[29] Renouf, M., Marmet, C., Guy, P., Fraering, A. L., et al., Nondairy creamer, but not milk, delays the appearance of coffee phenolic acid equivalents in human plasma. J Nutr 2010, 140, 259-263. [30] Stalmach, A., Steiling, H., Williamson, G., Crozier, A., Bioavailability of chlorogenic acids following acute ingestion of coffee by humans with an ileostomy. Archives of Biochemistry and Biophysics 2010, 501, 98-105.

[31] Guerra, A., Folesani, G., Mena, P., Ticinesi, A., et al., Hippuric acid in 24 h urine collections as a biomarker of fruits and vegetables intake in kidney stone formers. Int J Food Sci Nutr 2014, 65, 10331038.

[32] Lang, R., Dieminger, N., Beusch, A., Lee, Y. M., et al., Bioappearance and pharmacokinetics of bioactives upon coffee consumption. Analytical and Bioanalytical Chemistry 2013, 405, 8487-8503.

[33] Cho, K. O., Jo, Y. J., Song, B. K., Oh, J. W., Kim, Y. S., Colon transit time according to physical activity and characteristics in south korean adults. World Journal of Gastroenterology 2013, 19, 550555.

[34] Crozier, A., Del Rio, D., Clifford, M. N., Bioavailability of dietary flavonoids and phenolic compounds. Mol Aspects Med 2010, 31, 446-467.

[35] Rosa, N. N., Aura, A. M., Saulnier, L., Holopainen-Mantila, U., et al., Effects of disintegration on in vitro fermentation and conversion patterns of wheat aleurone in a metabolical colon model. $J$ Agric Food Chem 2013, 61, 5805-5816.

[36] Nordlund, E., Aura, A. M., Mattila, I., Kössö, T., et al., Formation of phenolic microbial metabolites and short-chain fatty acids from rye, wheat, and oat bran and their fractions in the metabolical in vitro colon model. Journal of Agricultural and Food Chemistry 2012, 60, 8134-8145. [37] Del Rio, D., Calani, L., Cordero, C., Salvatore, S., et al., Bioavailability and catabolism of green tea flavan-3-ols in humans. Nutrition 2010, 26, 1110-1116.

[38] Pekkinen, J., Rosa, N. N., Savolainen, O. I., Keski-Rahkonen, P., et al., Disintegration of wheat aleurone structure has an impact on the bioavailability of phenolic compounds and other phytochemicals as evidenced by altered urinary metabolite profile of diet-induced obese mice. Nutr Metab (Lond) 2014, 11, 1.

[39] Nørskov, N. P., Hedemann, M. S., Theil, P. K., Fomsgaard, I. S., et al., Phenolic acids from wheat show different absorption profiles in plasma: A model experiment with catheterized pigs. Journal of Agricultural and Food Chemistry 2013, 61, 8842-8850.

[40] Kern, S. M., Bennett, R. N., Mellon, F. A., Kroon, P. A., Garcia-Conesa, M. T., Absorption of hydroxycinnamates in humans after high-bran cereal consumption. Journal of Agricultural and Food Chemistry 2003, 51, 6050-6055.

[41] Anson, N. M., Selinheimo, E., Havenaar, R., Aura, A. M., et al., Bioprocessing of wheat bran improves in vitro bioaccessibility and colonic metabolism of phenolic compounds. J Agric Food Chem 2009, 57, 6148-6155.

[42] Rodriguez-Mateos, A., Vauzour, D., Krueger, C. G., Shanmuganayagam, D., et al., Bioavailability, bioactivity and impact on health of dietary flavonoids and related compounds: an update. Arch Toxicol 2014, 88, 1803-1853. 


\section{TABLE 1}

Nutritional values and ferulic acid content per portion of testing meals.

\begin{tabular}{cccc}
\hline & $\begin{array}{c}\text { WGB } \\
\text { Wholegrain Bread } \\
\mathbf{( 9 4} \mathbf{~ g )}\end{array}$ & $\begin{array}{c}\text { AB-94 } \\
\text { Aleurone-Enriched } \\
\text { Bread }\end{array}$ & $\begin{array}{c}\text { AB-190 } \\
\text { Aleurone-Enriched } \\
\text { Bread } \\
\mathbf{( 9 4} \mathbf{~ g )}\end{array}$ \\
\hline Energy (kcal) & 246 & 245 & $\mathbf{( 1 9 0 ~ g )}$ \\
Energy (kJ) & 1035 & 1035 & 496 \\
Fats (g) & 5.5 & 3.9 & 2092 \\
Carbohydrates (g) & 35.3 & 40.7 & 8.0 \\
Fiber (g) & 7.5 & 5.6 & 82.3 \\
Proteins (g) & 10.2 & 8.9 & 11.4 \\
Ferulic Acid (mg) & 87 & 43 & 18.0 \\
\hline
\end{tabular}

\section{TABLE 2}

Standard compounds used for metabolite quantification.
Standard compounds used for quantification

This article is protected by copyright. All rights reserved. 


\begin{tabular}{ll}
\hline $\begin{array}{l}\text { 3',4'-Dihydroxyphenylacetic acid } \\
\text { Hippuric acid }\end{array}$ & $\begin{array}{l}\text { 3' }^{\prime}, 4^{\prime} \text {-Dihydroxyphenylacetic acid } \\
\text { 4-Hydroxyhippuric acid }\end{array}$ \\
$\begin{array}{l}\text { Hydroxyhippuric acid } \\
\text { Hydroxybenzoic acid sulfate }\end{array}$ & 4-Hydroxyhippuric acid \\
3-(Phenyl)propionic acid-O- & Dihydrocaffeic acid-3-O-sulfate \\
sulfate & Dihydrocaffeic acid-3-O-sulfate \\
Vanillic acid-4-O-sulfate & Dihydroferulic acid-4'-O-sulfate \\
Feruloylglycine & Feruloylglycine \\
Dihydrocaffeic acid-3-O-sulfate & Dihydrocaffeic acid-3-O-sulfate \\
Ferulic acid-4'-O-sulfate & Ferulic acid-4'-O-sulfate \\
Dihydroferulic acid-4'-O-sulfate & Dihydroferulic acid-4'-O-sulfate \\
Sinapic acid- $O$-sulfate & Ferulic acid-4'-O-sulfate \\
Dihydroferulic acid-O-glucuronide & Dihydro(iso)ferulic acid-3-O-glucuronide \\
\hline
\end{tabular}

\section{TABLE 3}

Spectrometric characteristics used for metabolite quantification.

\begin{tabular}{|c|c|c|c|c|c|}
\hline Metabolites & RT & $\begin{array}{l}{[\mathrm{M}-\mathrm{H}]^{-}} \\
(\mathrm{m} / \mathrm{z})\end{array}$ & $\mathrm{Ms}^{2}$ & $\mathrm{MS}^{3}$ & $\begin{array}{l}\text { Sample } \\
\text { Detection }\end{array}$ \\
\hline $3^{\prime}, 4^{\prime}$-Dihydroxyphenylacetic acid & 2.06 & 167 & 123 & & Urine \\
\hline Hippuric acid & 2.75 & 178 & 134 & & Urine, Plasma \\
\hline 4'- Hydroxyhippuric acid & 1.93 & 194 & 100 & & \\
\hline Hydroxyhippuric acid & 2.17 & 194 & 150 & & Urıne, Plasma \\
\hline Hydroxybenzoic acid sulfate & 2.52 & 217 & 137 & 93,137 & Urine \\
\hline 3-(Phenyl)propionic acid-O-sulfate & 2.88 & 245 & 165 & 147,121 & Urine \\
\hline Vanillic acid-4-O-sulfate & 2.78 & 247 & 167 & $123,152,108$ & Urine \\
\hline Feruloylglycine & 3.69 & 250 & $\begin{array}{c}206,100,149 \\
175,191\end{array}$ & & Urine \\
\hline Dihydrocaffeic acid-3-O-sulfate & 2.55 & 261 & 181 & 137,119 & Urine \\
\hline Ferulic acid-4'-O-sulfate & 3.53 & 273 & 193 & $149,178,134$ & Urine, Plasma \\
\hline Dihydroferulic acid-4'-O-sulfate & 2.94 & 275 & 195 & $151,136,180,177$ & Urine, Plasma \\
\hline Sinapic acid-O-sulfate & 3.10 & 303 & 223 & 208, 179, 191 & Urine \\
\hline Dihydroferulic acid-O-glucuronide & 3.14 & 371 & 195 & $119,151,149,136$ & Urine \\
\hline
\end{tabular}

This article is protected by copyright. All rights reserved. 


\section{FIGURE 2}

Cumulative urinary excretion of ferulic acid metabolites: a) Ferulic acid-4'-O-sulfate, b)

Dihydroferulic acid-4'-O-sulfate, c) Dihydroferulic acid-O-glucuronide, and d) Feruloylglycine.

Values are expressed as cumulative $\mu$ mol excreted \pm SEM $(n=15)$. Different letters mean

significant differences comparing the three consumed product. A general linear model for repeated measures (ANOVA, LSD post-hoc test) was used to compare each metabolite among the three treatments. Statistical significance was set at $p<0.05$. WGB consists of $94 \mathrm{~g}$ of wholegrain bread, containing approximately $87 \mathrm{mg}$ of total ferulic acid; $A B-94$ consists of $94 \mathrm{~g}$ of a commercial bread enriched in aleurone fraction, containing approximately $43 \mathrm{mg}$ of total ferulic acid; AB-190 consists of $190 \mathrm{~g}$ of a commercial bread enriched in aleurone fraction, containing approximately $87 \mathrm{mg}$ of total ferulic acid.

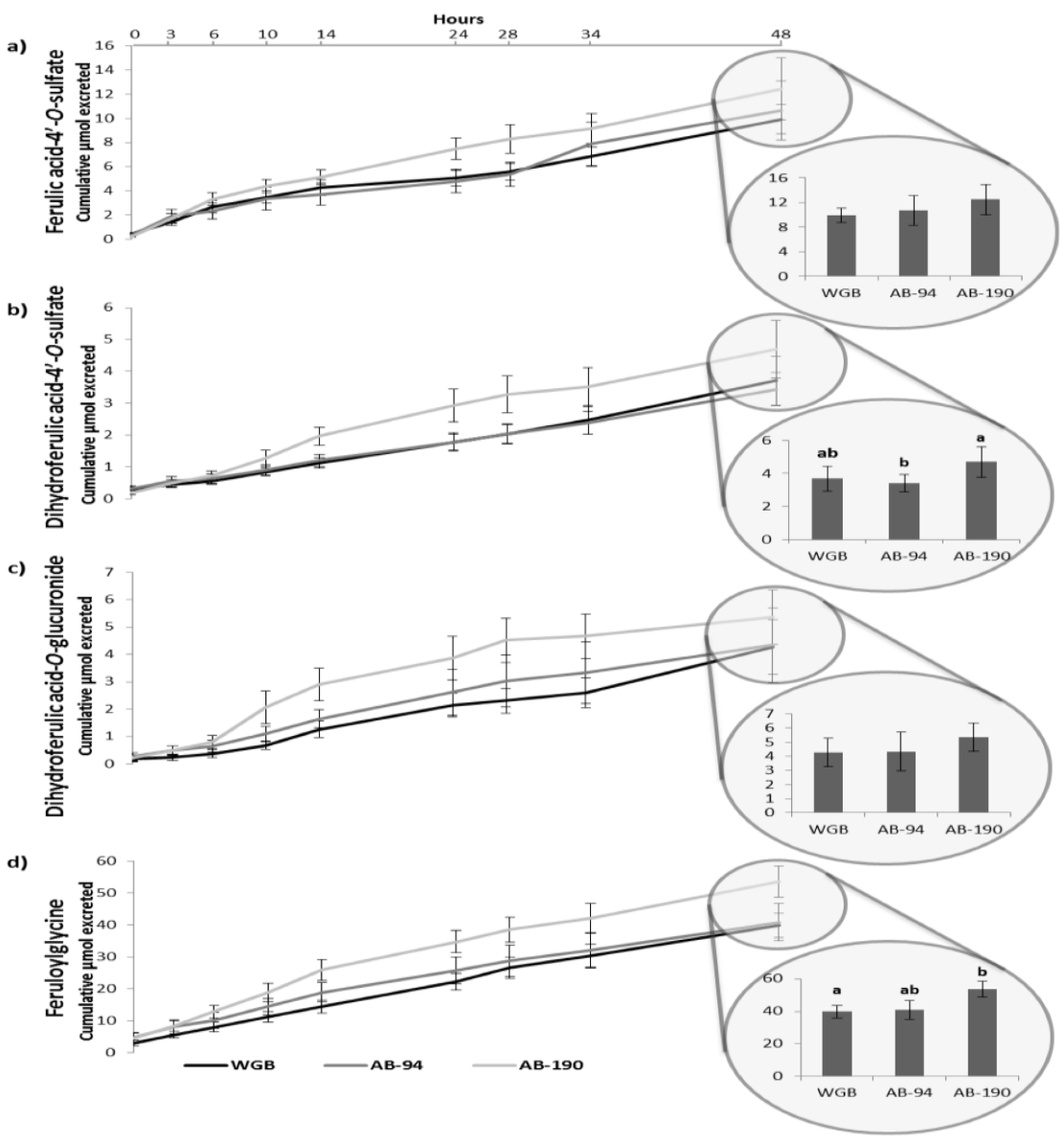

This article is protected by copyright. All rights reserved. 


\section{FIGURE 3}

Cumulative excretion of phenolic acid metabolites: a) Dihydrocaffeic acid-3-O-sulfate, b)

Sinapic acid sulfate, c) Vanillic acid-4-O-sulfate, and d) Hydroxybenzoic acid sulfate. Values are expressed as cumulative $\mu$ mol excreted \pm SEM $(n=15)$. Different letters mean significant differences comparing the three consumed product. A general linear model for repeated measures (ANOVA, LSD post-hoc test) was used to compare each metabolite among the three treatments. Statistical significance was set at $p<0.05$. WGB consists of $94 \mathrm{~g}$ of wholegrain bread, containing approximately $87 \mathrm{mg}$ of total ferulic acid; AB-94 consists of 94 $\mathrm{g}$ of a commercial bread enriched in aleurone fraction, containing approximately $43 \mathrm{mg}$ of total ferulic acid; AB-190 consists of $190 \mathrm{~g}$ of a commercial bread enriched in aleurone fraction, containing approximately $87 \mathrm{mg}$ of total ferulic acid.

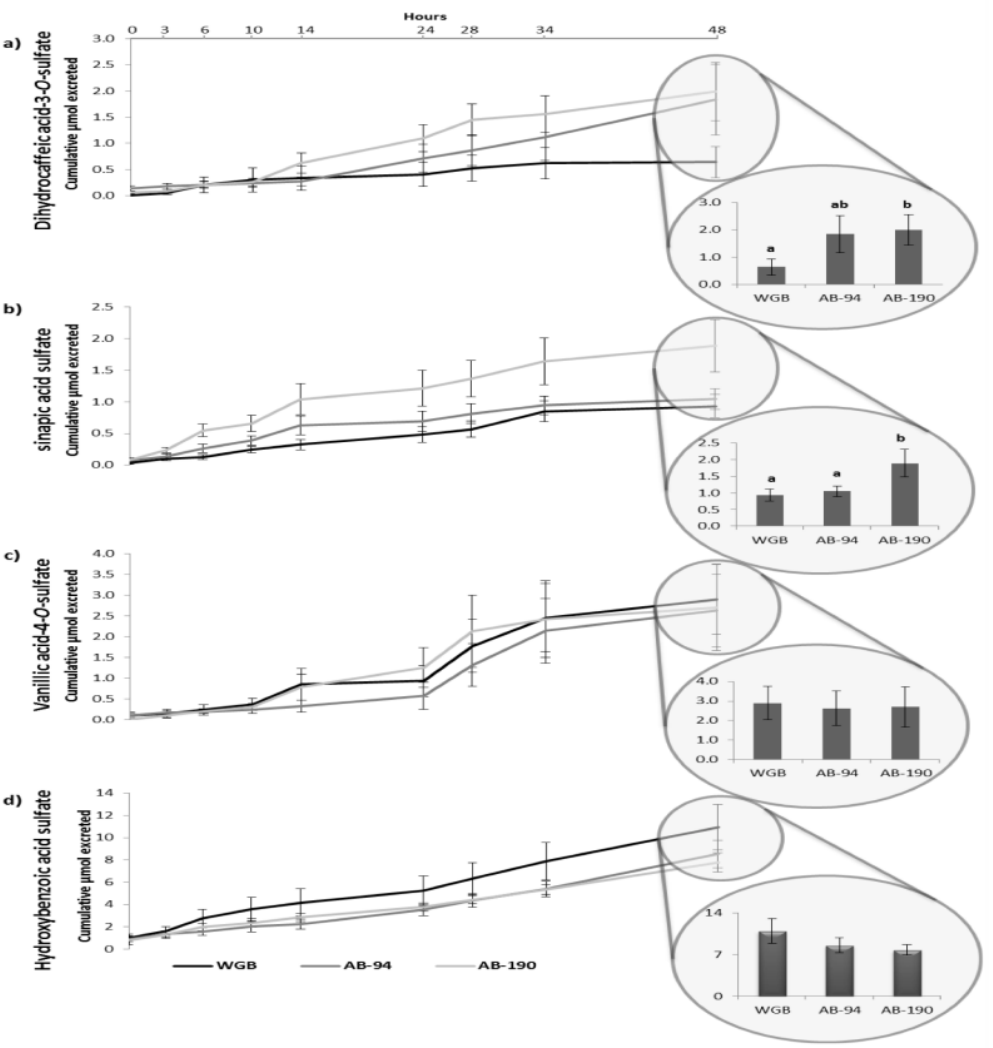




\section{FIGURE 4}

Ferulic acid bioavailability calculated comparing the total amount of ferulic acid consumed

by the 15 volunteers and the quantity excreted through its main metabolites, namely ferulic acid-4'-O-sulfate, dihydroferulic acid-4'-O-sulfate and dihydroferulic acid-O-glucuronide.

Different letters mean significant differences comparing the three consumed product. A general linear model for repeated measures (ANOVA, LSD post-hoc test) was used to compare the bioavailability $(p<0.05)$. WGB consists of $94 \mathrm{~g}$ of wholegrain bread, containing approximately $87 \mathrm{mg}$ of total ferulic acid; AB-94 consists of $94 \mathrm{~g}$ of a commercial bread enriched in aleurone fraction, containing approximately $43 \mathrm{mg}$ of total ferulic acid; AB-190 consists of $190 \mathrm{~g}$ of a commercial bread enriched in aleurone fraction, containing approximately $87 \mathrm{mg}$ of total ferulic acid.

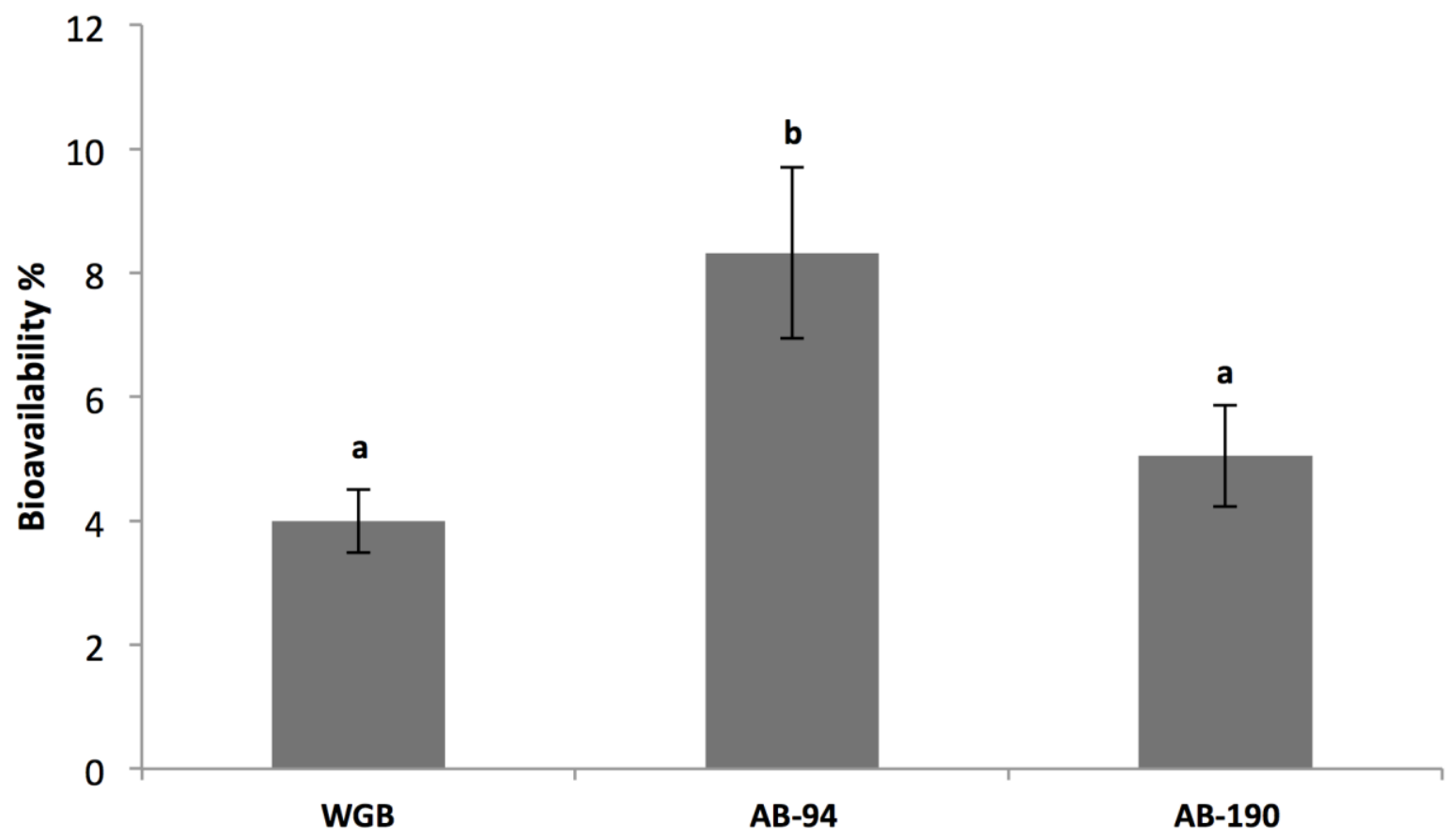

602 


\section{FIGURE 5}

Absorption kinetics of the phenolic metabolites detected in plasma. a) Ferulic acid-4' $-O$ sulfate, and b) Dihydroferulic acid-4'-O-sulfate. Values are expressed as $n m o l / L \pm S E M(n=$ 5). A general linear model for repeated measures (ANOVA, LSD post-hoc test) was used to compare pharmacokinetic parameters among the three treatments. Statistical significance was set at $p<0.05$. No statistically significant difference was observed. WGB consists of $94 \mathrm{~g}$ of wholegrain bread, containing approximately $87 \mathrm{mg}$ of total ferulic acid; AB-94 consists of $94 \mathrm{~g}$ of a commercial bread enriched in aleurone fraction, containing approximately $43 \mathrm{mg}$ of total ferulic acid; AB-190 consists of $190 \mathrm{~g}$ of a commercial bread enriched in aleurone fraction, containing approximately $87 \mathrm{mg}$ of total ferulic acid.
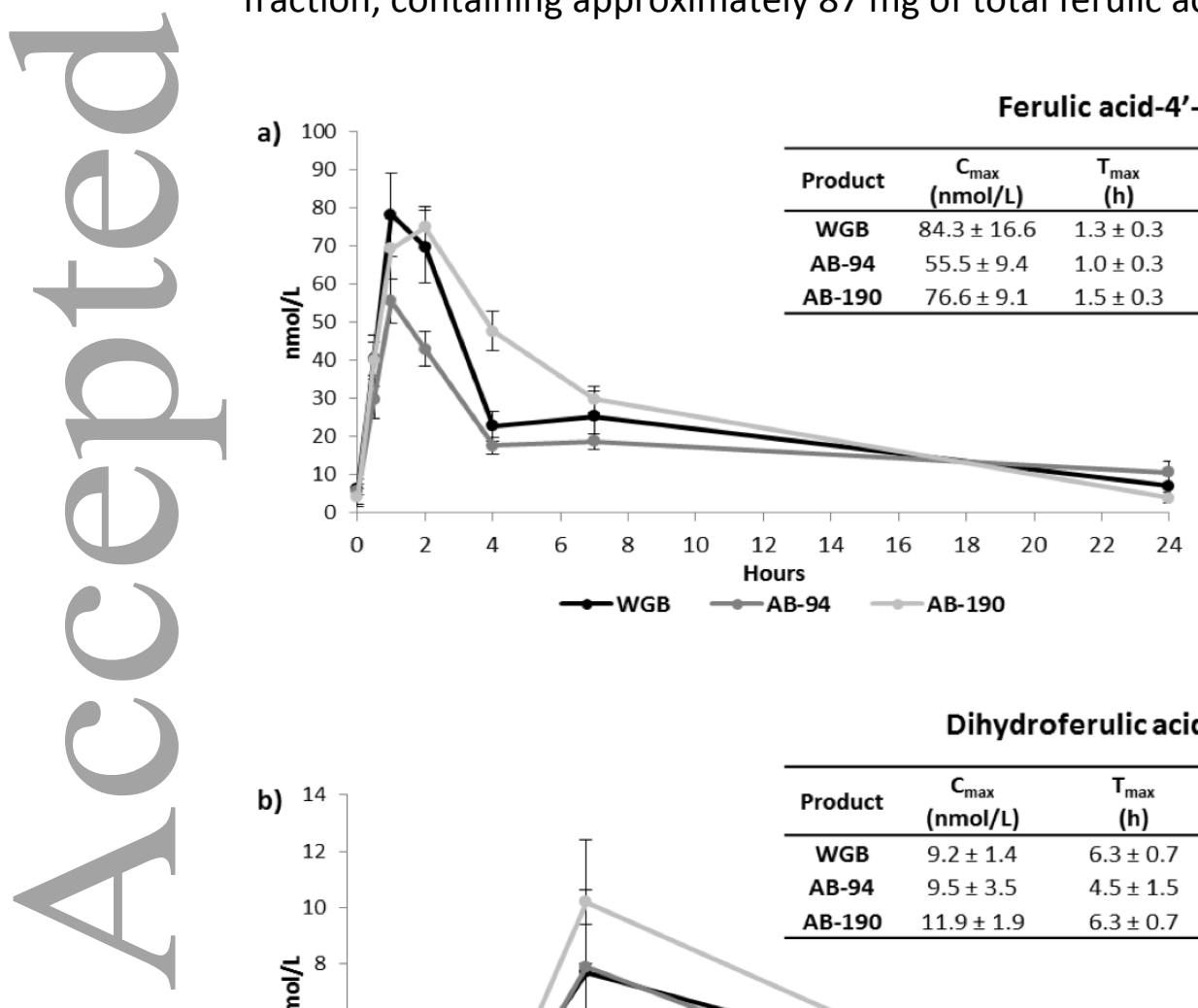

\section{Dihydroferulic acid-4'-O-sulfate}

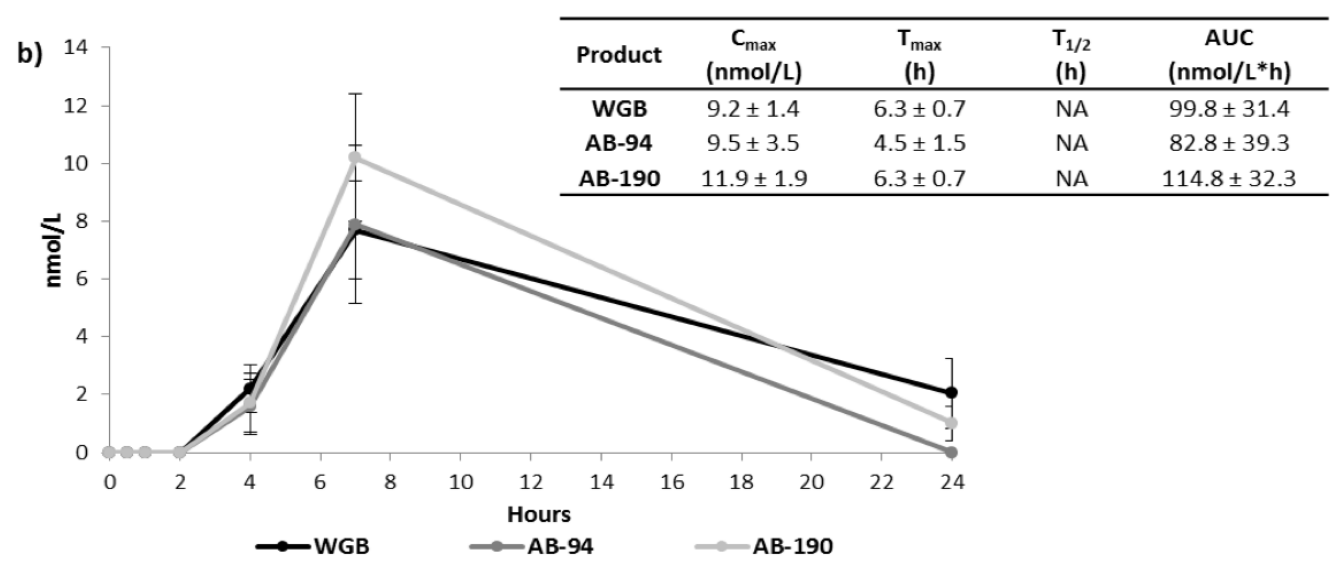

